\title{
Optimizing Nasal Interface for Continuous Positive Airway Pressure in Neonates
}

\author{
DEEPaK Chawla \\ Department of Pediatrics, Government Medical College Hospital, Chandigarh, India. \\ drdeepak@gmch.gov.in
}

$\mathrm{N}$ asal continuous positive airway pressure (CPAP) as the primary mode of respiratory support in preterm neonates with respiratory distress is associated with reduced needs of surfactant therapy and invasive ventilation, and improved survival without bronchopulmonary dysplasia [1,2]. Despite convincing evidence of benefits, use and success rate of CPAP remain variable across different neonatal units. Major challenges in CPAP application in routine clinical practice include ensuring proper fixation and avoiding nasal injury.

In comparison to nasopharyngeal or single nasal prongs, short binasal prongs are associated with lower incidence of CPAP failure [3]. However, art of fixation of short binasal prongs requires balancing the chance of frequent displacement likely with a loosely fitting prong, and the risk of nasal injury likely with a tightly fitting prong. Search for a better nasal interface has resulted in emergence of nasal mask as an alternative CPAP interface. However, evidence on relative efficacy of nasal prongs and nasal mask is sparse [4]. The randomized controlled trial by Goel, et al. [5], published in this issue of Indian Pediatrics, compares nasal mask and nasal prongs for CPAP delivery in neonates born at less than 34 weeks of gestation. Although, the rate of CPAP failure was halved in the nasal mask group, this difference did not reach statistical significance due to assumptions made during sample size calculation. CPAP failure rate of $40 \%$ with nasal prongs which was used for sample size calculation was much higher than $25-30 \%$ rate reported in literature or actually observed in the nasal prong arm of the study. Incidence of nasal injury was lower in the nasal mask group. However, pressure points and pattern of nasal trauma observed with nasal mask may be different from the injury to columella and nasal septa observed with nasal prongs. Application of nasal mask is more likely to cause pressure on nasal bridge and the junction of nasal septum and philtrum [4]. Assessing nasal skin status only at nares may miss the trauma caused by the nasal mask. Previous studies have reported equal incidence of nasal injury with both the interfaces $[4,6]$. A recent study has reported lower incidence of nasal injury on rotating the use of nasal prong and nasal mask than on isolated use of each interface [7].

Success of CPAP also depends on the actual distending pressure delivered to the lungs. With bubble CPAP, the pressure delivered depends on the gas flow and leakage at the nasal interface. Higher flows needed to achieve bubbling in the nasal mask group indicates greater leakage at the nasal interface in comparison to nasal prongs. This may be the reason behind lower incidence of air leaks observed in the nasal mask group of the study. This also highlights the need to monitor the actual pressure delivered at the nose with CPAP. Current bubble CPAP devices lack inbuilt pressure manometers making this task difficult. Till medical device regulators and manufacturers take the corrective steps, it is imperative for the end users to use standalone manometers to monitor the real-time pressure delivered with CPAP.

Whatever nasal interface is used, success of CPAP depends on achieving accurate and consistent pressure delivery and minimizing the iatrogenic nasal injury. Therefore, one needs to find the perfectly fitting interface for each neonate. It is almost impossible for device manufactures to build different sizes and shapes which can fit unique anatomy of each neonate. This means that manufacturing of CPAP interface needs to be individualized. Personalized CPAP nasal mask as a concept of proof has been shown to improve CPAP effectiveness in children with obstructive sleep apnea and craniofacial anomalies [8]. Availability of on-site threedimensional (3-D) spatial anatomy profiler and 3-D printer in near future can realize the prospect of having a custom-built perfect-fit CPAP interface for each neonate.

Funding: None; Competing interests: None stated.

\section{REFERENCES}

1. Ho C. Using a spectral reflectance technique to measure transcutaneous bilirubin in neonates: A new device. Issues 
EDITORIAL

Emerg Health Technol. 2002;33:1-4.

2. Schmölzer GM, Kumar M, Pichler G, Aziz K, O'Reilly M, Cheung PY. Non-invasive versus invasive respiratory support in preterm infants at birth: Systematic review and meta-analysis. BMJ. 2013;347:f5980.

3. De Paoli AG, Davis PG, Faber B, Morley CJ. Devices and pressure sources for administration of nasal continuous positive airway pressure (NCPAP) in preterm neonates. Cochrane Database Syst Rev. 2008;1:CD002977

4. Kieran EA, Twomey AR, Molloy EJ, Murphy JFA, O'Donnell CPF. Randomized trial of prongs or mask for nasal continuous positive airway pressure in preterm infants. Pediatrics. 2012;130:e1170-6.

5. Goel S, Mondkar J, Panchal H, Hegde D, Utture A, Manerkar S. Nasal mask versus nasal prongs for delivering nasal continuous positive airway pressure in preterm infants with respiratory distress: A randomized controlled trial. Indian Pediatr. 2015;52:1035-40.

6. Yong S, Chen S, Boo N. Incidence of nasal trauma associated with nasal prong versus nasal mask during continuous positive airway pressure treatment in very low birthweight infants: A randomised control study. Arch Dis Child Fetal Neonatal Ed. 2005;90:F480-3.

7. Newnam KM, McGrath JM, Salyer J, Estes T, Jallo N, Bass WT. A comparative effectiveness study of continuous positive airway pressure-related skin breakdown when using different nasal interfaces in the extremely low birth weight neonate. Appl Nurs Res. 2015;28:36-41.

8. Aufieri R, Picone S, Gente M, Paolillo P. 3D printing in neonatal care. Ital J Pediatr. 2015;41:A1. 